\title{
Creación de una línea de transporte fluvial en el río Bobonaza desde puerto Latasas hasta Sarayacu, bajo la modalidad de un subsidio porcentual
}

\author{
Salomón Jaya Quezada \\ Director del Centro de Excelencia en Transporte Intermodal y Fluvial-CETIF \\ jayasalo@yahoo.com \\ Mónica Coronel Silva \\ Investigadora del Centro de Excelencia en Transporte Intermodal y Fluvial-CETIF \\ xelamon7@hotmail.com
}

Recibido: 16 de octubre / Aprobado: 21 de diciembre 2017

\section{Resumen}

Esta propuesta se realizó con el fin de brindar a las poblaciones asentadas en las orillas del río Bobonaza, provincia de Pastaza-Ecuador un servicio de transporte oportuno y subsidiado que contribuya con su desarrollo y bienestar. Para esto nos hemos basado en varios criterios técnicos respecto a la reducción de tiempos del transporte, a la minimización de riesgos en la navegabilidad; se realizó un diagnóstico del servicio de transporte fluvial en la ruta y se hizo un análisis de la oferta y demanda. El tramo en estudio cubre la ruta puerto Latasas - Saraya$\mathrm{cu}$, tomando en consideración que en la actualidad no existe una línea fija que atienda al sector.

Palabras clave: transporte fluvial, Latasas, Sarayaku, Bobonaza. 


\begin{abstract}
Abastract
This proposal was made in order to provide the populations settled on the banks of the Bobonaza River, Province of Pastaza-Ecuador, a timely and subsidized transportation service that contributes to their development and well-being. For this we have based on several technical criteria regarding the reduction of transportation times, to minimize risks in navigability; a diagnosis of the fluvial transport service was made on the route and an analysis of supply and demand was made. The section under study that covers the Latasas - Sarayacu port route, taking into account that currently there is no fixed line that covers the sector.
\end{abstract}

Keywords: river transport, Latasas, Sarayaku, Bobonasa. 


\section{Introducción}

continuación se presenta los resultados del proyecto: "Creación de una línea
de transporte fluvial en el río Bobonaza desde el puerto Latazas hasta Sara-
yacu, bajo la modalidad de un subsidio porcentual"; realizado por el Centro de Excelencia en Transporte Intermodal y Fluvial (CETIF) y la Universidad Central del Ecuador, para brindar el servicio de transporte fluvial a las poblaciones asentadas en las orillas del río Bobonaza en la provincia de Pastaza-Ecuador, con el fin de mejorar el desarrollo y bienestar de las comunidades.

\section{Objetivos:}

\section{General}

Realizar el estudio de una línea de transporte fluvial eficiente, segura y de acceso a todas las personas, mediante un subsidio porcentual de la tarifa establecida, para los habitantes de la cuenca del Bobonaza, que sea ambientalmente amigable con la naturaleza, que esté en concordancia con el desarrollo de la zona donde se implementará, que cumpla con los preceptos de ser un transporte sostenible, social y económico con el uso racional de los recursos.

\section{Específicos}

- Realizar un diagnóstico del servicio de transporte fluvial en la ruta Latasas-Sarayacu con el fin de optimizar la cobertura y calidad de la movilidad fluvial en las riberas del río Bobonaza entre Latasas y Sarayacu.

- Realizar un estudio de oferta y demanda del uso del transporte fluvial.

- Presentar una propuesta técnica-económica de transporte fluvial con un subsidio porcentual.

\section{Alcance}

El desarrollo económico de la cuenca del río Bobonaza está íntimamente vinculado a las inversiones que se realicen en sus vías de comunicación como carreteras, puertos, aeropuertos, ríos y pistas de aterrizaje con el fin de desarrollar la intermodalidad en el transporte.

El transporte fluvial en esta cuenca es un factor muy importante en el ámbito social, económico y ambiental, especialmente en esta región donde la provisión de carreteras se dificulta por su geografía y por sus frágiles ecosistemas, por lo tanto, son los ríos la única vía de traslado en la zona.

El transporte es vital para dar un adecuado soporte al desarrollo económico y social donde los patrones actuales y tendencia no son sostenibles. 
El transporte sostenible salva vidas, genera condiciones para el desarrollo económico con equidad y protege al medio ambiente local y global.

Con la implementación de esta propuesta se mejoraría la calidad de vida de los pobladores que se encuentran en el área de influencia directa del estudio para lo cual se realizará un diagnóstico de la situación del transporte fluvial del sector, un análisis de oferta y demanda del servicio de transporte fluvial en el tramo en estudio desde puerto Latasas hasta Sarayacu.

\section{Ubicación del lugar de estudio}

El estudio está ubicado en la provincia de Pastaza, parroquia Canelos en el tramo Canelos - puerto Latasas - Sarayacu.

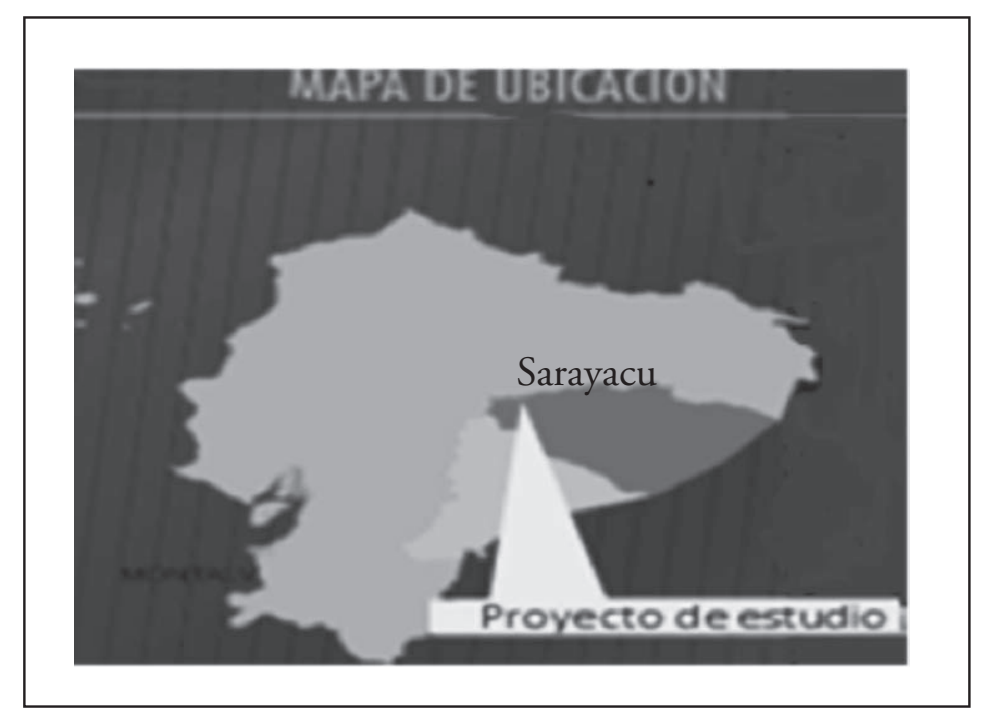

Esquema 1. Ubicación del proyecto.

Fuente: CETIF

El área de influencia se determinó en función de los flujos fluviales de transporte, tomando en consideración el origen y destino de los pasajeros, las embarcaciones y la carga movilizada esto permitió a su vez definir los centros de generación y recepción de los flujos de transporte fluvial, además se tomó en consideración la existencia de los diferentes medios de transporte que permiten el acceso de los flujos hacia las vias navegables como los ríos afluentes, y trochas carrozables. El área de influencia directa se determinó tomando en consideración los alcances de accesibilidad a las poblaciones asentadas a lo largo del río Bobonaza, esta área es de un kilómetro a cada lado del cauce desde el eje del río y corresponde a las poblaciones aledañas asentadas a lo largo del río Bobonaza. 


\section{Vialidad}

La parroquia Sarayacu, caracterizada por su ubicación geográfica particular, posee dos tipos de sistemas de movilidad claramente definidos, el aéreo y fluvial, y uno adicional, no tan definido, es el terrestre a nivel de senderos que conectan a las comunidades cercanas entre ellas. Los senderos son aperturas esporádicas que se realizan en búsqueda de nuevas alternativas de conexión.

El GAD parroquial de Sarayacu tiene un convenio firmado con el GAD provincial de Pastaza para la construcción de una carretera desde Latasas hasta Umupi de $24.5 \mathrm{~km}$. Las comunidades utilizarán esta vía para sacar sus productos agrícolas para lo cual necesitarán vehículos comunitarios, esta vía permitiría que se atienda las urgencias médicas y otros aspectos de importancia para la parroquia de Sarayacu.

El transporte desde Sarayacu es por medios fluviales, aéreos y terrestres como la vía Latasas - Pakayaku, la vía Llanchama-cocha - Moretecocha - Curiyacu hasta la vía El Triunfo - Arajuno, y la vía que conecta Mashient - Kintiuk - hasta la Chapintza en la parroquia Simón Bolívar.

Las conexiones por las redes fluviales alcanzan 242,17 km, tomando el Bobonaza como el eje central.

El sistema aéreo, implica trasladar pasajeros o cargamentos de un lugar a otro, mediante el uso de aviones, avionetas, helicópteros, es un sistema de transporte enfocado en la conectividad de la parroquia con otras jurisdicciones. Existen varias pistas aéreas que cumplen con el traslado de personas y cargamento hacia otras parroquias.

Sin embargo, las pistas son muy cortas, el piso no es el adecuado, pocas están lastradas, cuando llueve, lo cual es frecuente, se vuelven lodazales peligrosos para los aterrizajes. Estas pistas no tienen torres de control, ni siquiera una manga para el viento, la aproximación es completamente visual. Para Sarayacu el transporte aéreo es prioritario. Desde junio del 2014 opera la empresa Aerosarayacu que tiene dos avionetas Cessna T206H y 182P, para cinco y tres pasajeros, respectivamente. Fueron adquiridas por la comunidad en USD 390 mil, según información del diario El Comercio en 2016.

El sistema fluvial, comprende el transporte de pasajeros o carga a través del uso de canoas a motor y a sus palancas, que diariamente cumplen su labor en beneficio de la población a través de los ejes fluviales existentes.

Los problemas que enfrenta la comunidad respecto a movilidad son los senderos existentes, que no cuentan con mantenimiento, altos costos de transportación aérea en avionetas de 3 y 5 pasajeros que limitan el servicio, los traslados vía fluvial son temporalmente extensos, entre 6 y 8 horas dependiendo de las características del río, no existe carretera entre Latasas y Sarayacu.

\section{Tráfico local}

El movimiento tanto de personas como de carga y el comercio entre las poblaciones que se encuentran en el tramo Latasas - Sarayacu y su área de influencia directa 
e indirecta, se realiza con pequeńas embarcaciones, como canoas para un solo hombre, canoas medianas con motor fuera de borda y pekes pekes, entre otros.

\section{Definición del problema y sus causas}

\section{Definición del problema central}

El problema central que se ha identificado en el río Bobonaza es la falta de una línea de transporte fluvial permanente en el tramo Latasas - Sarayacu para que movilice a la población asentada en sus riberas durante todo el año, con costos acordes a la economía local.

\section{Causas que generan el problema central}

\section{Causas directas}

- Falta de estudios frecuentes de navegabilidad del río (el río Bobonaza o está muy seco o está muy lleno, entonces es un río muy peligroso).

- Falta de una empresa de transporte que movilice a la población.

\section{Causas indirectas}

- Inexistencia de datos estadísticos y mediciones hidrométricas en el río.

- Inexistencia de sistemas de señalización y ayudas a la navegación.

- Inexistencia de monitoreo a las restricciones a la navegación, como por ejemplo, las grandes cantidades de piedra que existe en el lugar.

\section{Diagnóstico}

\section{Diagnóstico de la situación actual}

Actualmente las comunidades del tramo Latasas - Sarayacu no tienen una línea de transporte fija que preste un servicio continuo o por lo menos quincenal de movilidad fluvial, por esta razón la población utiliza el sistema de transporte aéreo, en época de estiaje, por la disminución del calado del río se puede apreciar varios malos pasos que restringen el transporte seguro.

\section{Obstáculos que restringen la navegación en la cuenca del río Bobonaza}

Para que la movilidad fluvial sea eficiente y segura en el tramo Latasas - Sarayacu es necesario eliminar ciertos obstáculos a la navegación, además de realizar constantes estudios de las variaciones estacionales del río, su morfología, señalización del canal navegable, estudios hidrológicos e hidráulicos del río.

Entre los principales obstáculos que restringen la navegación se tienen:

Palizadas: Se presentan en épocas de crecida; se observa troncos de árboles y arbustos flotando a la deriva en los cauces del río, desplazándose debido a las corrientes que se producen por el incremento de caudal. La caída de árboles al río puede ser 
por varias causas como por la erosión de las riberas, por la gravedad y condiciones de equilibro comienzan a inclinarse hasta caer, por la acción y destrucción del tallo que los sostiene, por estrangulamiento producido por otras formas de vida, etc.

Erosión de riberas: Se produce por la alta velocidad en crecida del río, tanto en los tramos rectos como en las concavidades.

Sedimentación y formación de playas de arena: La sedimentación es un proceso natural donde las partículas de suelo erosionado se depositan en las partes convexas del cauce, obligando al río a erosionar su lado opuesto.

La gran cantidad de piedras que existen aguas abajo de Latasas son un verdadero obstáculo en temporada de sequía puesto que al bajar el nivel de las aguas estas piedras restringen los calados de las embarcaciones que pueden pasar sobre ellos.

Meandros: Se presentan en la parte baja, no presentan dificultades a la navegación en este río.

Señalización fluvial y ayuda a la navegación: La señalización fluvial no existe, puesto que este río no está en una escala de ser hidrovía, pero debe ponerse ciertas referencias en los mayores peligros.

Falta de datos estadísticos y mediciones hidrométricas: En la actualidad el río Bobonaza no cuenta con datos estadísticos que permitan registrar su comportamiento en las diferentes épocas del año, tales como cambios en los niveles y caudales, transporte de sedimentos, y batimetrías en épocas de estiaje y de crecida.

\section{Sectores económicos}

El sistema económico de la parroquia Sarayacu enfrenta problemas en cuanto a la producción por falta de transporte seguro por el río, no se conoce la producción agrícola de acuerdo a la aptitud de los suelos. En cuanto a los casos de emigración estos son temporales al igual que en los otros recintos como Latasas, Sarayacu, etc.

\section{Actividad agrícola}

La producción agrícola de las comunidades es para la alimentación de cada familia. La forma de producción es en chacras o huertos familiares. La comercialización de los productos agrícolas es mínima, su rentabilidad es baja debido a los altos costos de transporte que se generan por las distancias.

\section{Actividades turísticas}

No existen referencias sobre atractivos turísticos a lo largo del río, sin embargo, su riqueza natural y étnica hacen de todo su territorio un lugar atractivo para compartir con el mundo. 
La venta de artesanías y cerámicas, es mínima en todos los poblados.

La presencia de las nacionalidades Kichwa, Achuar, y Sápara en su territorio, con formas únicas de convivencia comunitaria y marcadas de una cosmovisión propia, permiten sugerir como alternativa de ingresos económicos el ecoturismo.

La presencia de bosques, cascadas, playas de los ríos, saladeros, ríos y vertientes propios de la morfología convierten a su territorio en potencial turístico a explotarse.

Existe la operadora de turismo Papangu Tours que funciona en el Puyo, ésta se dedica al turismo comunitario en la parroquia de Sarayacu.

\section{Formulación}

\section{Análisis de la oferta y demanda}

Las carreteras que unen la ciudad del Puyo con Latasas son de primero, segundo y tercer orden y en algunos casos se evidencia ciertas dificultades en el acceso, principalmente el tramo Canelos-Puerto Latasas. El transporte de pasajeros desde el Puyo hasta puerto Canelos lo realiza la cooperativa de buses Centinela del Cóndor, a un costo de US\$ 1.60 hasta el puente de Canelos, esta cooperativa mediante un convenio con camionetas complementa el servicio hasta Latasas a un costo de US\$ 0.90. En Latasas no existe una terminal que permita el arribo de las unidades, y su principal problema es el mal estado de la vía.

Puerto Latasas representa el punto más importante de transferencia tanto de carga como de pasajeros que recibe o entrega para los diferentes destinos a lo largo del río Bobonaza.

El "embarcadero" en puerto Latasas no cuenta con infraestructura básica, ni regulaciones, ni normas de seguridad para la movilidad fluvial de personas y carga.

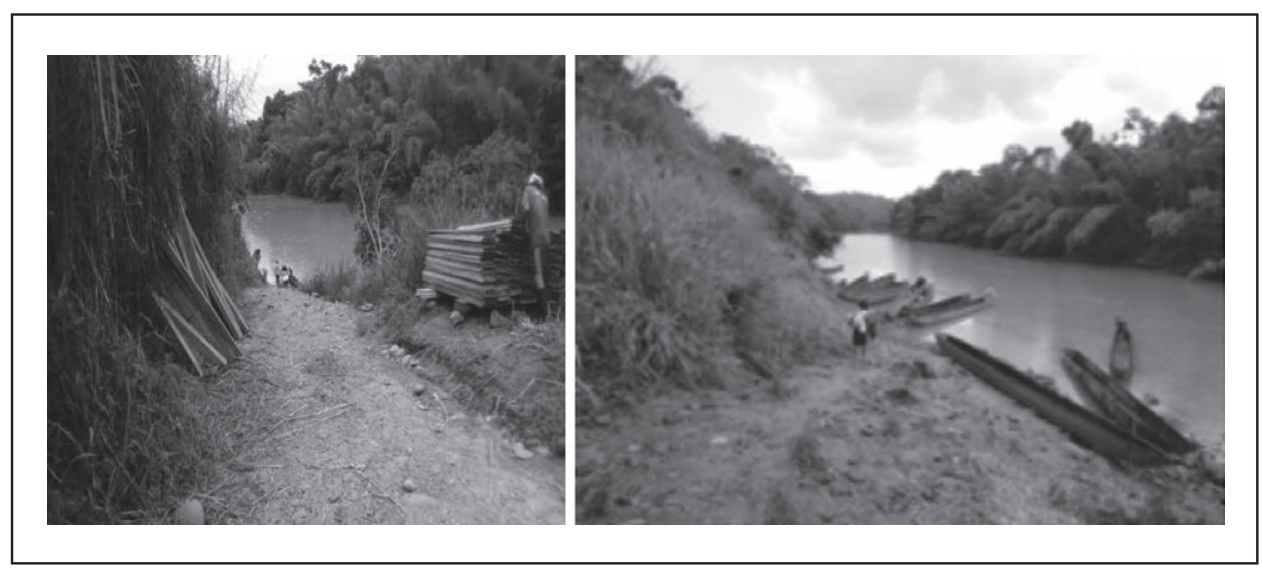

Fotografías 1 y 2. Embarcadero en puerto Latasas.

Fuente: CETIF 
El estado de la ruta fluvial en el tramo Latasas-Sarayacu presenta varios problemas de palizadas en época de crecida y pasos peligrosos en época de estiaje, estos y otros factores dificultan una navegabilidad segura.

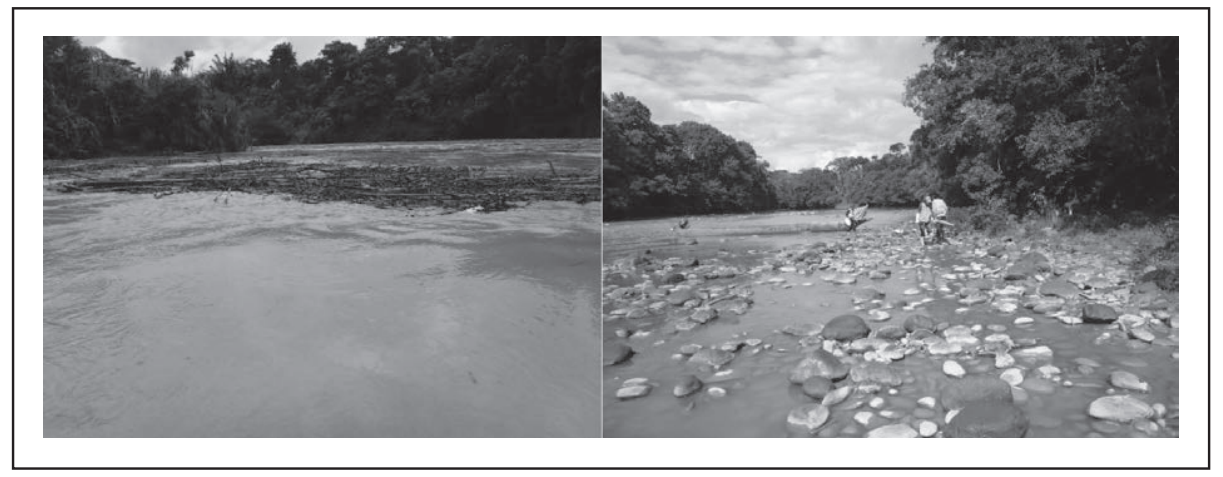

Fotografías 3 y 4. Palizada y presencia de piedras en el río Bobonaza.

\section{Fuente: CETIF}

El acceso a la parroquia de Sarayacu se realiza de dos maneras, vía aérea desde el aeropuerto de la Shell y fluvial por medio del río Bobonaza, desde puerto Latasas que se encuentra a dos horas por tierra del Puyo, esto se realiza en canoa a motor, se tarda aproximadamente entre cinco a seis horas dependiendo de las condiciones del río.

La oferta de transporte fluvial desde Latasas hacia la comunidad de Sarayacu es mínima.

La demanda del transporte fluvial está en función de las necesidades de la población y comunidad de Sarayacu. Según investigaciones previas realizadas por el CETIF se encontró que para la parroquia de Sarayacu la frecuencia de movilización de las comunidades es de 231 personas mensualmente. Por lo que será necesario brindar un servicio de transporte fluvial que cubra la demanda existente en el sector.

El objetivo de la aplicación de un subsidio porcentual de transporte para los habitantes de la ribera del río Bobonaza hasta Sarayacu es reducir los costos de los productos básicos y mejorar las condiciones de vida de personas de estos sectores, brindarles mejores oportunidades para desarrollarse e integrarse debido a que por el alto costo del transporte se encarecen los mismos en esos lugares, las instituciones gubernamentales o no gubernamentales serían las que fijen la cobertura del subsidio para las comunidades a movilizarse.

La distancia del tramo fluvial entre puerto Latasas y Sarayacu es de 67 kilómetros. El combustible necesario para la movilización desde Latasas hasta la comunidad de Sarayacu en época de crecida es de 12 galones y en temporada de estiaje se consume 20 galones (USD 17,76) y en temporada de estiaje se consume 20 galones (USD 29,60) en promedio en el recorrido de ida y vuelta se consume 40 galones 
(USD 59.20), el precio del galón de combustible extra es de USD 1.48. (Precio de la gasolina en Ecuador es 0.39 centavos por litro) ${ }^{1}$.

Tabla 1. Consumo de combustible entre Latasas-Sarayacu

\begin{tabular}{|c|c|c|c|c|c|}
\hline \multirow[b]{2}{*}{ Ruta } & \multicolumn{5}{|c|}{$\begin{array}{cc}\text { Distancia } & \begin{array}{c}\text { Consumo combustible } \\
\text { (galones) }\end{array} \\
\end{array}$} \\
\hline & km & Crecida & Estiaje & $\begin{array}{c}\text { Costo de } \\
\text { galón de } \\
\text { combustible } \\
\text { UDS }\end{array}$ & $\begin{array}{c}\text { Costo total de } \\
\text { combustible } \\
\text { USD }\end{array}$ \\
\hline Latasas-Sarayacu & 67 & 12 & & 1,48 & 17,76 \\
\hline \multirow[t]{2}{*}{ Latasas-Sarayacu } & 67 & & 20 & 1,48 & 29,6 \\
\hline & & \multicolumn{2}{|c|}{$\begin{array}{c}\text { Consumo Combustible } \\
\text { Promedio (galones) }\end{array}$} & & \\
\hline Latasas-Sarayacu & 134 & & & 1,48 & 59,2 \\
\hline
\end{tabular}

Fuente: CETIF

Para cubrir la demanda serán necesarias dos lanchas con un motor fuera de borda de $25 \mathrm{Hp}$ que se movilicen desde Latasas hasta Sarayacu y viceversa. Las canoas a utilizarse podrán ser dos de las que dispone la comunidad de Sarayacu.

Actualmente la movilización desde Latasas hasta Sarayacu tiene un costo de USD 15 este es un precio sumamente alto para la comunidad, tomando en cuenta la situación económica en la que se desarrollan, pues según las investigaciones del CETIF su situación de pobreza es casi extrema, además considerando que si su frecuencia de salida fuera semanal esto significa un gasto de USD120 mensuales de ida y retorno y, la tarifa establecida en la ruta Puyo-Canelos-Puerto Latasas y viceversa es de USD 2,50.

Las frecuencias u horarios que se encuentran establecidos para prestar el servicio de transporte terrestre público intracantonal de pasajeros en la ruta Puyo-Canelos-Puerto Latasas y viceversa son los siguientes:

Tabla. 2. Frecuencias establecidas

\begin{tabular}{|l|c|}
\hline \multicolumn{1}{|c|}{ RUTA } & FRECUENCIAS U HORARIOS \\
\hline PUYO-CANELOS-PUERTO LATASAS & $06: 00,06: 30,09: 30,12: 30,14: 30,16: 30$ Y 18:30 \\
\hline PUERTO LATASAS-CANELOS-PUYO & $06: 00,07: 00,08: 30,09: 00,13: 00,16: 00$ Y 18:00 \\
\hline
\end{tabular}

Fuente: ANTP

1 http://es.globalpetrolprices.com/Ecuador/gasoline_prices/ 
Por esta razón, se concluye que es necesario la aplicación de un subsidio porcentual para el transporte fluvial en este tramo, pues la comunidad de Sarayacu necesita movilizarse por trabajos, comercio, salud, estudios.

El subsidio sería únicamente para los habitantes de esta región; la tasa que se define es del $50 \%$ con el fin de incrementar el desarrollo sustentable de la población de Sarayacu y de las poblaciones ribereñas del Bobonaza.

Este subsidio complementará el faltante de poder adquisitivo para realizar la adquisición de un bien o servicio por parte del ciudadano. Actualmente en el país se subsidia diversos productos y servicios, como el gas, la gasolina, el diésel y el transporte.

\section{Estimación de la demanda potencial}

Según las encuestas realizadas por el CETIF en la población de Sarayacu existen 4.626 habitantes distribuidos en 891 familias, de las cuales se estima un 26\% como la cantidad de personas que demandan el servicio de transporte fluvial mensualmente, lo que corresponde a 231 pasajeros, o 2.772 demandantes por año. (PDOT, Sarayacu 2015).

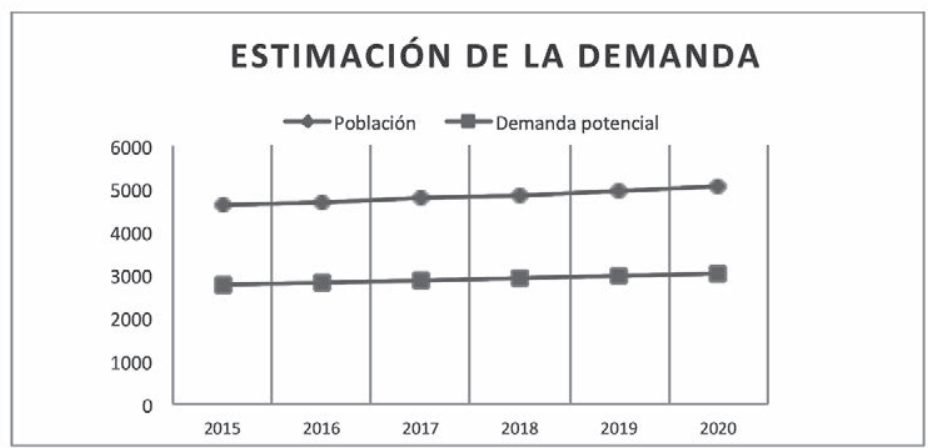

Gráfico 1. Estimación de la demanda.

Fuente: CETIF

\section{Proyección de ingresos}

Para realizar la proyección de ingresos se tomó en cuenta la demanda potencial del servicio de transporte fluvial a brindarse, estimada de acuerdo a cada ańo y por un horizonte de 5 ańos, y a un precio estimado y subsidiado de USD 7,50 por pasajero.

Esta demanda potencial se proyectó desde 2015 hasta 2020 con y sin subsidio, dándonos un ingreso total sin subsidio para el año 2020 de USD 105.829,35 y con subsidio USD 52.914,67 


\section{Presupuesto de inversión}

La inversión para la puesta en marcha del proyecto se encuentra definida como el monto de recursos necesarios para la ejecución de las actividades afines al proyecto. La inversión total se encuentra distribuida entre activos fijos, diferidos y el capital de trabajo necesarios para la operación del proyecto, sumando un total de USD 22.881 .00

El monto inicial a invertirse se encuentra distribuido así: el 50\% en capital de trabajo, el 30\% como activos fijos y el 20\% restante como inversiones diferidas.

El presupuesto de inversión sumando inversiones fijas más inversiones diferidas y capital de trabajo es de USD 22,881.00.

Como inversiones fijas está la construcción de oficinas de administración debidamente equipadas en Latasas y Sarayacu para ofrecer el servicio de expendio de boletos, información y logística del viajero.

\section{Proyección de costos}

Los costos anuales durante el horizonte del proyecto se encuentran estimados de acuerdo a la tasa de inflación. Estos costos están comprendidos por costos fijos como salarios, depreciaciones y amortizaciones dándonos un total de USD 13,479.00 y costos variables como suministros de oficina y servicios básicos un promedio de USD 1.179.46, dándonos un promedio de gastos totales de USD $14.658,46$.

\section{Flujo neto de caja}

La inversión inicial del proyecto de USD 27.601,00 se recuperaría en el segundo ańo de su implementación USD 32.231,36, lo que demuestra que a pesar de ser un transporte subsidiado es rentable financieramente y sobre todo es un gran apoyo para los habitantes de la región.

\section{Fuentes de financiamiento}

El gobierno parroquial de Sarayacu junto con el gobierno provincial de Pastaza serían los encargados de definir las fuentes de financiamiento. Al igual que el subsidio propuesto del 50\% para el servicio de transporte fluvial entre Latasas y Sarayacu por el río Bobonaza; por supuesto, deben realizar un análisis consensuado como proyecto definitivo para la creación de la línea de transporte.

\section{Alianzas estratégicas}

Las alianzas estratégicas son importantes que se establezcan entre la comunidad de Sarayacu y los actores públicos y privados para mejorar las condiciones de vida de la población integrándoles a través del transporte fluvial para lograr un desarrollo sustentable de la región. 


\section{Evaluación}

\section{Beneficios del proyecto}

- Salud: Permitirá el acceso de la población a los programas preventivos de salud $y$, a los enfermos asistir oportunamente a los puestos de salud, localizados en los centros urbanos; asimismo, se logrará que los profesionales de la salud, puedan llegar al área de influencia de esta propuesta con relativa oportunidad.

- Educación: Facilitará el acceso de la población a centros especializados de capacitación localizados en las cabeceras parroquiales o cantonales, permitirá el acceso de los educadores a las áreas en donde se encuentra la población.

- Cultura: La integración de las comunidades de la cuenca del Bobonaza facilitará la expansión y conservación de su cultura propia y permitirá el acceso a programas culturales de mayor relevancia.

- Comercio: Se verá favorecido y desarrollado cuando se tenga una navegación y un transporte oportuno y económico; sin transporte no hay comercio; por lo tanto, si se quiere mejorar los niveles de productividad, es necesario propiciar y mejorar las condiciones del transporte fluvial.

- Turismo: Se ha incorporado en esta propuesta el turismo comunitario como estrategia de producción económica en algunas comunidades, considerando que esta actividad puede ser una alternativa para el desarrollo del sector, dada la riqueza de sus recursos naturales, paisajísticos y culturales que disponen.

\section{Ventajas y desventajas del proyecto}

Es conveniente analizar las ventajas y desventajas que ofrecería la creación de una línea de transporte fluvial permanente en el río Bobonaza.

Ventaja: Un transporte fluvial permanente, oportuno, seguro y con costo reducido.

Desventajas: No poder atender volúmenes grandes de carga y pasajeros por el tipo de embarcaciones adaptadas a las características propias del río y la hidrología de su cuenca.

\section{Conclusiones y recomendaciones}

- Este estudio permitirá mejorar el escaso servicio de transporte fluvial en el río Bobonaza en el tramo Latasas y Sarayacu que hace que los habitantes de la región no exploten sus recursos y por ende no mejoren su situación de vida.

- La implementación de esta propuesta reactivará el sector productivo, turístico y comercial, generando un desarrollo económico sostenible para los habitantes de la región.

- Para los efectos de la habilitación de una empresa de transporte público fluvial, se requiere que exista la demanda del servicio de pasajeros y de carga. Tener la infraestructura adecuada para el funcionamiento de la empresa con sus sucursales operativas a lo largo del río Bobonaza en el tramo Latasas-Sarayacu. 
- Es necesario el apoyo financiero del Gobiernos Autónomo Descentralizado Provincial de Pastaza, el Gobierno Autónomo Descentralizado Cantonal de Pastaza, entes gubernamentales y gobiernos locales para el mejoramiento e implementación de la infraestructura portuaria básica, embarcaderos y equipos de servicios portuarios tanto en el sector de puerto Latasas como en las comunidades del eje.

- Es conveniente tener una permanente preocupación por el mejoramiento y mantenimiento de las condiciones de navegabilidad del río Bobonaza, especialmente en las épocas de estiaje, que es la época donde se presentan sectores críticos o malos pasos que restringen la navegación segura.

- Cabe recomendar que en fuertes crecidas del río no se debe navegar y también en las noches.

\section{Referencias}

ANT-Pastaza. (2014). Alcance al estudio de necesidades de transporte público intracantonal e intraprovincial de la provincia de Pastaza, Ecuador.

Argenis Heredia. (Mayo 2016). Integración del servicio de transporte terrestre público intracantonal de pasajeros al transporte fluvial en la ruta puyo-Canelos-puerto Latasas, del cantón Pastaza. Quito.

GAD Cantonal de Pastaza. (2011). Plan de Desarrollo y Ordenamiento Territorial del Cantón Pastaza 2011-2025.

Ministerio de Transporte. Habilitación de empresas de transporte fluvial, https:// www.nomasfilas.gov.co/memoficha-tramite/-/tramite/T5433

Superintendencia de Puertos y Transporte, República de Colombia, Yolima Paredes Morato, Bogotá, julio de 2010.

UCE, CTT, CETIF. (2012). Estudio de navegabilidad del río Bobonaza desde Canelos Puerto Latasas, hasta Sarayacu - Primera Etapa, Pastaza, Ecuador. 